\title{
Rendezvous in Higher Dimensions
}

\author{
Steve Alpern ${ }^{1}$ and Vic Baston ${ }^{1,2}$ \\ ${ }^{1}$ Department of Mathematics \\ The London School of Economics \\ London WC2A 2AE, U.K. \\ ${ }^{2}$ Department of Mathematics \\ University of Southampton \\ Southampton, U.K. \\ Supported by NATO grant PST.CLG.976391
}

April 30, 2004 


\begin{abstract}
Two players are placed on the integer lattice $Z^{n}$ (consisting of points in $n$ dimensional space with all coordinates integers) so that their vector difference is of length 2 and parallel to one of the axes. Their aim is to move to an adjacent node in each period, so that they meet (occupy same node) in least expected time $R(n)$, called the Rendezvous Value. We assume they have no common notion of directions or orientations (i.e., no common notion of 'clockwise'). We extend the known result $R(1)=3.25$ of Alpern and Gal to obtain $R(2)=197 / 32=$ 6.16 , and the bounds $2 n \leq R(n) \leq\left(32 n^{3}+12 n^{2}-2 n-3\right) / 12 n^{2}$. For $n=2$ we characterize the set of all optimal strategies and show that none of them simultaneously maximize the probability of meeting by time $t$ for all $t$. This behaviour differs from that found by Anderson and Fekete, and the authors, for the related problem where the players are initially placed at diagonals of one of the squares of the lattice $Z^{2}$.
\end{abstract}




\section{Introduction}

This paper generalizes the fixed-distance version of the linear rendezvous problem introduced in [7] to higher dimensions. That problem asks how two unitspeed players placed an initial fixed distance $D$ apart on the line, and faced in random directions (which they each call 'forward'), can meet in minimum expected time. Neither knows the direction to the other, nor do they have a common notion of a 'positive' or 'forward' direction. By taking $D=2$ we may model the line as the lattice $Z=Z^{1}$ of integers, with the players moving to adjacent nodes (integers) in each integer period, until the first (meeting) time that they occupy the same node. (Taking $D$ even ensures they will not pass each other on the line without meeting.) A strategy for each player can be taken as a sequence of $F$ 's (for Forward) and B's (for Backward), which determines a players motion relative to his starting node and his initial direction. Adopting Player I's coordinate system, with his initial node as 0 and his forward direction to the right, we equiprobably place Player II at the nodes -2 and +2 . We may consider that there are four agents of Player II, two starting at -2 (facing in either direction) and two at +2 . It was shown in [7] that the strategy pair

$$
(F, F, B, B, B, B) \text { (forPlayer I) and }(F, B, B, B, F, F) \text { (for II) }
$$

is optimal, having minimum expected meeting time 13/4 (called the rendezvous value and, since the line has dimension $n=1$, denoted here $R(1)=13 / 4=$ $3.25)$. It is easy to verify the expected meeting time $13 / 4$ for this strategy pair (though establishing optimality is obviously harder). If the two players start facing each other (that is, Player II starts at +2 facing left), then since they both start with a forward move and meet at time $t=1$ at node +1 . This corresponds to the upper right entry in the meeting time matrix for the four agents of Player II, and the reader should verify the other three. The expected meeting time is therefore $(2+1+6+4) / 4=13 / 4$.

\begin{tabular}{|c|c|c|}
\hline location $\backslash$ direction & $\rightarrow$ & $\leftarrow$ \\
\hline+2 & 2 & 1 \\
\hline-2 & 6 & 4 \\
\hline
\end{tabular}

The optimal strategy (1) for $\Gamma$ (1) is almost a Wait For Mommy (WFM) strategy in which Player I optimally searches out the possible initial starting points of II, while II stays still. In fact $(F, F, B, B, B, B)$ is such a Mommy strategy, though II does not Wait. However, note that II is indeed back at his starting node at the two times $t=2,6$ that I might search it. At the intermediate time $t=4$, Player II searches out a possible starting point of I. So we may call this strategy an Alternating Wait For Mommy (AWFM) Strategy, in which the players alternate taking the role of Mommy and searching out starting points of the other. Such strategies will play a role in the higher dimensional games $\Gamma(n)$. (The analysis of more general linear rendezvous problems can be found in [13] and [6].)

To generalize this problem to $n$ dimensions, we consider the game $\Gamma(n)$ in which the two players are initially placed in the lattice $Z^{n}$ ( $n$-vectors of integers, 
with nodes adjacent if they differ by 1 in one coordinate and are identical in the others) so that their vector difference is of length 2 and parallel to one of the coordinate axes. We assume that they don't know the initial location of the other, and that they have no common ordering of the axes (in particular, no common notion of clockwise for the planar problem, $n=2$ ). The organization and main results of the paper are as follows.

In Section 2, we give a rigorous definition of the rendezvous problem on the planar lattice $Z^{2}$, for a general distribution of the initial vector between the players. The presentation is similar but simpler than that given in [4] and contains some general optimality conditions.

In Section 3 we solve the game $\Gamma(2)$, establishing (Theorem 14) that the rendezvous value is $R(2)=197 / 32$ (here there are 32 agents of Player II, rather than the 4 in the case $n=1$, see the table 18). Although the AWFM strategy is not optimal, a variation of it called the Nearly Alternating Wait For Mommy (NAWFM)Strategy (drawn in Figure 4) is optimal. We also determine the full set of optimal strategies (Theorem 15). We show that no strategy is uniformly optimal, in the sense that it simultaneously maximizes the probability of meeting by time $t$, for all $t$. We also show that the players can do better (lower expected meeting time) if they are have a common notion of clockwise, though the best they can do in that case is a further problem which we solve in a subsequent paper [5]. These results may be contrasted with those for the planar rendezvous model introduced by Anderson and Fekete [10] and extended by the authors [4], for the diagonal start problem (players start at opposite diagonals of a square in the $Z^{2}$ lattice). In that model all optimal strategies are also uniformly optimal and having a common notion of clockwise does not help the players.

In Section 4, we obtain (Theorem 16) bounds on the rendezvous value $R(n)$, showing that is asymptotically bounded above by $8 n / 3$. These results are the first obtained for rendezvous in more than two dimensions. The results obtained here are reported in the book [8], which attributes them to an earlier version of this paper.

The version of rendezvous search adopted in this paper is the so called playerasymmetric (or just asymmetric) version, in which the players can have distinct strategies. For example, they may have mobile phones and agree which role each will take, e.g. one Mommy and one Child in the WFM strategy pair. The other, player-symmetric version (where they both must adopt the same mixed strategy) will not be discussed here, but can be found in [1], [9], and [11].

A general survey of the rendezvous search problem can be found in [2] and a unified presentation results up to 2003 can be found in Book II of [8]. The main works on planar rendezvous are [10] and [15].

\section{Rendezvous in the Plane: strategies and agents}

In this section we give a formal presentation of rendezvous on the planar lattice $Z^{2}$ without a common notion of clockwise. A similar but more general presentation is given in [4]. The (lattice) distance $d$ between two nodes is de- 
fined as the sum of the edges in a shortest connecting path, or equivalently $d\left(\left(z_{1}, z_{2}\right),\left(w_{1}, w_{2}\right)\right)=\left|z_{1}-w_{1}\right|+\left|z_{2}-w_{2}\right|$. At time $t=0$ Nature places the two players on even nodes with the vector $v$ from I to II drawn from a given distribution. (A node $z \in Z^{2}$ is called even if the sum of its coordinates is even; otherwise it is called odd.) In every time period each player must move to an adjacent node. This restriction, combined with the 'even distance' initial placement (originating in the interval network of Howard [14]) ensures that the two players will always have the same parity, and cannot pass each other on an edge without meeting at a node.

We analyze the progress of the game in terms of Player I's coordinate system (and sense of clockwise). In this perspective, the initial random placement is achieved by Nature placing I at the origin facing North $(\mathcal{N})$ and placing II at the even node $v_{i}$, with probability $p_{i}, i=1, \ldots, K$, facing equiprobably in either of the four possible directions, and with either the same or opposite notion of clockwise as Player I. Aside from this section, dealing with arbitrary initial locations $v_{i}$, we will be mainly concerned with the following initial placement.

Definition 1 The 'parallel' game $\Gamma_{P}$ begins with the initial distribution in which the initial displacement vector between the players has length 2 and is parallel to one of the coordinate axes. Equivalently, $I$ is initially placed at the origin $(0,0)$ and II is initially placed equiprobably at one of the four nodes $v_{1}=(0,2)$, $v_{2}=(2,0), v_{3}=(0,-2), v_{4}=(-2,0)$.

When the game begins, the players have no common notion of locations or directions in the plane, and no common notion of clockwise. As observers, we adopt I's coordinate system. The orientations of Player II can be seen as transformations (or rigid motions, or symmetries) of the 'standard orientation' of Player I. Figure 1 shows the eight equiprobable orientations.

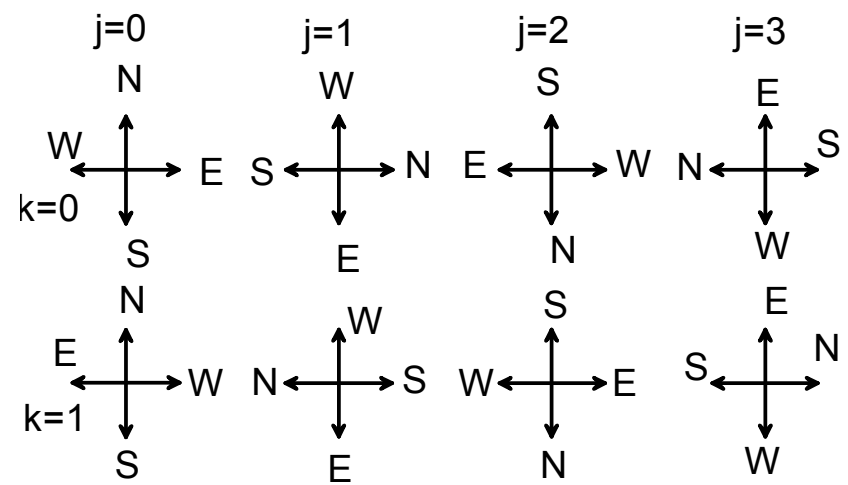

Figure 1: Eight orientations in the plane.

The orientations in the top row $(k=0)$ all have the same notion of clockwise as Player I (the usual one), with the upper left orientation the standard one, and the one in column $j$ obtained from it by applying the $90^{\circ}$ clockwise rotation 
$\mathcal{R}$ to it $j$ times. The orientations in the bottom row $(k=2)$ have the other (reversed) notion of clockwise, and can be obtained from the one above by a single application of the reflection $\phi$ about the vertical axis. Formally,

$$
\begin{aligned}
\mathcal{R}^{j} & =\text { clockwise rotation by angle } j \pi / 2, j=0,1,2,3, \\
\phi\left(w_{1}, w_{2}\right) & =\left(-w_{1}, w_{2}\right), \text { and the eight orientations of II are } \\
\phi^{k} \mathcal{R}^{j}, j & =0,1,2,3 ; k=0,1
\end{aligned}
$$

The orientations determine how Player II will move when given an instruction (strategy). For example, if the instruction is for II to go $E$ (East) then (in Player I's coordinate system, which we also adopt as observers) he will go $S$ (South) if he has orientation $j=1, k=0$; and he will go $N$ if he has orientation $j=3, k=1$.

We can now define a strategy and show how a pair of strategies determines a set of meeting times for the two players, one for each initial configuration (initial location $v_{i}$ and initial orientation $\left.j, k\right)$.. We begin by allowing the null move $(0,0)$, but we will remove this possibility later.

Definition 2 A strategy for a player (in either game $\Gamma^{C}$ or $\Gamma$ ) is a sequence of directions $D_{t} \in\{N=(1,0), E=(1,0), S=(0,-1), W=(-1,0)\}, t=1,2, \ldots$ - A player pursuing this strategy moves successively one unit in his direction $D_{0}, D_{1}, \ldots$, according to his initial orientation. Equivalently, it can be seen as his net displacement $f(t)$ at time $t$ from his initial location, given by $f(0)=(0,0)$ and for $t \geq 1$,

$$
f(t)=\sum_{m=1}^{t} D_{m} .
$$

So for example the strategy beginning $N, E, E$, (I's strategy, thick grey line in Figure 2) corresponds to a net displacement function $f$ with

$$
[f(0), f(1), f(2), f(3)]=[(0,0),(0,1),(1,1),(2,1)] .
$$

We shall deal with strategy pairs $(f, g)$ where Player I adopts $f$ and II adopts $g$. Sometimes we will use the symmetric notation $\left(f_{1}, f_{2}\right)$. In this setting, the location of Player I at time $t$ is simply $f(t)$, while the location of II (in I's coordinate system) depends on his initial configuration, as described below. If the initial configuration $(i, j, k)$ gives Player II initial location $v_{i}$ and orientation $\phi^{k} \mathcal{R}^{j}$, then the location of Player II at time $t$ under strategy $g$ is given by

$$
g_{i, j, k}(t)=v_{i}+\phi^{k} \mathcal{R}^{j}(g(t)) .
$$

Definition 3 The $8 K$ (in our example, 32) paths $g_{i, j, k}$ are called the agents of Player II. We call $g_{i, j, k}$ the agent starting at $v_{i}$ in direction $j$, with the same (if $k=0$ ) or different (if $k=1$ ) notion of clockwise as $I$. Each agent from $v_{i}$ is the actual path of player II with probability $p_{i} / 4$. We will sometimes use the simpler two subscript agent notation $g_{i, j}=g_{i, j, 0}$ and $g_{i, j}^{\prime}=g_{1, j, 1}$ 
The time taken for agent $g_{i, j, k}$ to be met by Player I is called its meeting time, and denoted

$$
\omega_{i, j, k}(f, g)=\min \left\{t: f(t)=g_{i, j, k}(t)\right\},
$$

and the time required to meet all the agents is called maximum time $M(f, g)$, where

$$
M(f, g)=\max _{i, j, k} \omega_{i, j, k}(f, g) .
$$

For each time $t$ we calculate the number of agents $x_{t}$ that Player I meets (for the first time) at time $t$ as

$$
x_{t}=x_{t}(f, g)=\#\left\{(i, j, k): \omega_{i, j, k}(f, g)=t\right\},
$$

and we call the sequence $\left[x_{1}, x_{2}, \ldots, x_{M}\right]$ the agent number profile, or sometimes just agent profile, or just profile.

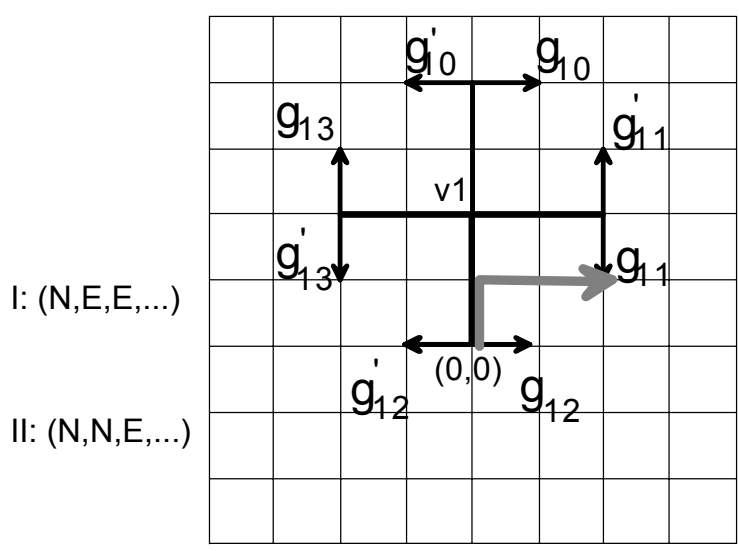

Figure 2: Meetings at $t \leq 3$, for $(\mathrm{N}, \mathrm{E}, \mathrm{E}),(\mathrm{N}, \mathrm{N}, \mathrm{E})$

Figure 2 illustrates several important concepts for the case where the strategy pair begins with $(\mathrm{N}, \mathrm{E}, \mathrm{E}, \ldots)$ for I and $(\mathrm{N}, \mathrm{N}, \mathrm{E}, \ldots)$ for II.. Player I's path $(0,0),(0,1),(1,1),(1,2)$ for $t \leq 3$ is shown in a thick grey line. The possible paths taken by II if he starts at $v_{1}=(0,2)$, that is, the 8 paths $g_{1, j, k}(t)$, $j=0, \ldots 3, k=0,1$, are drawn in medium black lines starting at $v_{1}=(0,2)$. Note that all the paths $g_{1, j, 0}(t)=g_{i, j}(t)$ in our notation make right turns at time 3, while the $g_{1, j}^{\prime}=g_{1, j, 1}$ make left turns. Note that Player I meets agents $g_{1,2}^{\prime}$ and $g_{1,2}$ at node $(0,1)$ at time 1 , and meets agent $g_{1,1}$ at node $(2,1)$ at time 3 . Thus in our notation we have $\omega_{1,2,0}=\omega_{1,2,1}=1$ and $\omega_{1,1,0}=3$. Note that when there are no turns since the last return to a starting point, agents $g_{i, j}$ and $g_{i, j}^{\prime}$ will be at the same location, so if one is met, the other will be also. Since it 
can be seen that Player I will not meet agents from any other starting nodes $v_{i}$ by time 3 , this means that Player I meets two agents at time $t=1$, no agents at time $t=2$, and one agent at time $t=3$, so that $x_{1}=2, x_{2}=0, x_{3}=1$, and the agent number profile begins $[2,0,1, \ldots]$. For comparison with a later table (18) we write this as a table of $\omega_{i, j, k}$, with blank entries depending on the strategy for $t>3$.

\begin{tabular}{|c|c|c|c|c|c|c|c|c|}
\hline$\omega_{i, j, k}$ & \multicolumn{4}{|l|}{$k=0$} & \multicolumn{4}{|l|}{$k=1$} \\
\hline$j$ & 0 & 1 & 2 & 3 & 0 & 1 & 2 & 3 \\
\hline$i=1$ & & 3 & 1 & & & & 1 & \\
\hline
\end{tabular}

Given a strategy pair $(f, g)$, the expected value of the meeting times $\omega_{i, j, k}$ is called the expected meeting time and denoted $T(f, g)$. Thus

$$
\begin{aligned}
T(f, g) & =\frac{1}{8 K} \sum_{i, j, k} p_{i} \omega_{i, j, k}(f, g), \quad \text { or simply } \\
& =\frac{1}{32} \sum_{i, j} \omega_{i, j}(f, g) \text { for parallel start }(K=4) .
\end{aligned}
$$

If $M=M(f, g)$ is finite, we may also calculate $T(f, g)$ by the meeting number sequence $x=\left[x_{1}, x_{2}, \ldots, x_{M}\right]$, as

$$
\begin{aligned}
T(f, g) & =\frac{1}{8 K} \sum_{t=1}^{M} t x_{t}, \text { or simply } \\
& =\frac{1}{32} \sum_{t=1}^{M} t x_{t}, \text { for parallel start }(K=4) .
\end{aligned}
$$

The rendezvous value $R$ for the game $\Gamma$ is the least expected time,

$$
R(\Gamma)=\min _{f, g} T(f, g),
$$

and any pair $f, g$ achieving the minimum is called optimal.

A stronger notion of optimality is the following.

Definition 4 A strategy pair is called uniformly optimal if for all $t$ it maximizes the probability that the players have met by time $t$. (Note that if there is a uniformly optimal strategy, then all optimal strategies must be uniformly optimal.)

A uniformly optimal strategy maximizes the expected utility $U(\omega)$ of the meeting time $\omega$ as long as the utility function $U$ is non-increasing in $\omega$ (earlier meetings are preferred to later ones); an optimal strategy is only required to accomplish this for the particular utility function $U(\omega)=-\omega$.

The authors have shown that, given the order in which the agents are met, any optimal strategy pair is greedy in the sense that next the agent is met 
as quickly as possible, with both players taking geodesics to the next meeting point. The following result was proved without the assumption that the players must move in each period (that is, staying still was allowed), and hence shows that our assumption here that they must move does not take away any optimal strategies.

Theorem 5 ([4]) Let $\left(f_{1}, f_{2}\right)$ be an optimal strategy pair. Define $\omega^{0}=0$ and let $\omega^{1}<\omega^{2}<\cdots<\omega^{K}$ denote the associated set of meeting times with the $8 K$ agents, listed in increasing order. Let d denote the graph distance on the lattice $Z^{2}$. Then

$$
d\left(f_{i}\left(\omega^{m-1}\right), f_{i}\left(\omega^{m}\right)\right)=\omega^{m}-\omega^{m-1}, \text { for } i=1,2 \text { and } m=1, \ldots, K .
$$

In other words, both players move in time-minimizing paths between consecutive meeting points. In particular, neither player ever stays still, and consequently both players are at even (odd) nodes at all even (odd) times. Furthermore, if the agent $g_{i, j, k}$ is met at time $\omega^{m}$, then that meeting point $f_{1}\left(\omega^{m}\right)=g_{i, j, k}\left(\omega^{m}\right)$ is a lattice midpoint of the locations of I and agent $g_{i, j, k}$ of II at time $\omega^{m-1}$, hence occurs at the earliest possible time (given their locations at time $\omega^{m-1}$ ).

\section{Parallel Start in the Plane}

In this section we study the parallel start game in the plane (Definition 1). From the perspective of the previous section (general starting nodes) this would be denoted $\Gamma_{P}$; from the perspective of the next section (general dimension $n$ ) this would be denoted $\Gamma(2)$, that is, $n=2$. We adopt the latter here. It will be convenient to introduce the notation $\mathcal{S}=\{(0,2),(2,0),(0,-2),(-2,0)\}$ for the set of Player II starting nodes, and $\mathrm{S}_{0}$ for the set $\mathcal{S}$ augmented by Player I's starting point $(0,0)$. Note that all nodes in $\mathcal{S}_{0}$ have even parity.

The main results of this section are Theorems 13, 14, and 15, which together determine the rendezvous value $R(\Gamma(2))=197 / 32$ and the complete set of optimal strategies for $\Gamma(2)$. Since we know players following optimal strategies will move in every period (never stay still), we will henceforth only consider strategies with this property. Since the players both start $\left(\right.$ in $\mathcal{S}_{0}$ ) at nodes of even parity, it follows that both are at even nodes at even times; at odd nodes at odd times.

\subsection{Special Strategies}

We now define some particular strategies, AWFM (valid for all $\Gamma(n)$ ) and NAWFM (for $\Gamma(2)$ ). In [8], a family of strategies called Alternating Wait For Mommy (AWFM) was proposed for the general parallel start game $\Gamma(n)$. The players alternate taking the role of Mommy (searching out the starting points of the other) and Baby (coming back to one's starting point to be found there when the other comes looking). We will use these strategies to analyze the $n$-dimensional case in the final section. 
Definition 6 A strategy in $\Gamma=\Gamma_{P}$ is called AWFM if Player I successively visits the $2 n$ possible starting locations of II (in any order) at times $\mathcal{T}_{1}=\{2,6$, $\ldots, 2+4(2 n-1)\}$, while returning to his start $(0,0)$ at the intermediate times $\mathcal{T}_{2}=\{4,8, \ldots, 4(2 n-1)\}$; and Player II makes his first move a single unit in any direction, is back at his start at times $\mathcal{T}_{1}$, and visits all but one of the possible initial locations of I at times $\mathcal{T}_{2}$. The maximum time for this strategy is clearly $M=2+4(2 n-1)$, or $M=14$ for $n=2$.

It was shown in [8] that, for $n=2$, any AWFM strategy (as illustrated below in Figure 3) gives the maximal probability of meeting by time $t$ for any $t \leq 7$, and suggested that this strategy might in fact be optimal (like the $n=1$ version, where optimality was established in [7]), or even uniformly optimal.

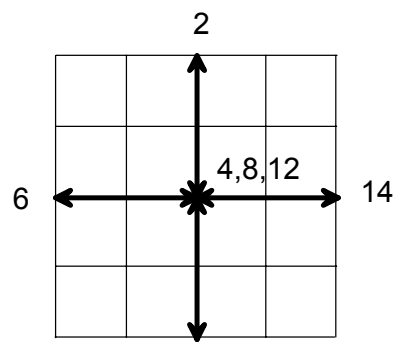

10

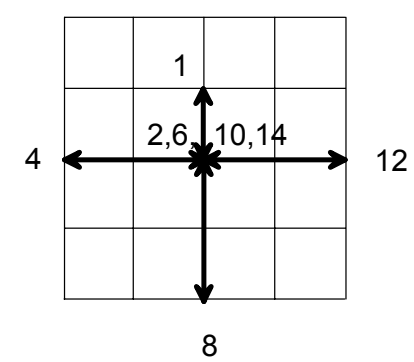

$(\mathrm{N}, \mathrm{N}, \mathrm{S}, \mathrm{S}, \mathrm{W}, \mathrm{W}, \mathrm{E}, \mathrm{E}, \mathrm{S}, \mathrm{S}, \mathrm{N}, \mathrm{N}, \mathrm{E}, \mathrm{E}) \quad(\mathrm{N}, \mathrm{S}, \mathrm{W}, \mathrm{W}, \mathrm{E}, \mathrm{E}, \mathrm{S}, \mathrm{S}, \mathrm{N}, \mathrm{N}, \mathrm{E}, \mathrm{E}, \mathrm{W}, \mathrm{W})$

Figure 3: An AWFM strategy $(\breve{f}, \breve{g})$ for $n=2$.

The meeting time sequence $x$ for $(\breve{f}, \breve{g})$ (and indeed for any AWFM strategy) is given by

$$
x=[2,6,0,6,0,6,0,4,0,4,0,2,0,2], \text { with } T(\breve{f}, \breve{g})=\frac{99}{16} .
$$

The strategy $(\tilde{f}, \tilde{g})$ drawn in Figure 4 has a lower expected meeting time $T$ than AWFM. Note that I's strategy $\tilde{f}$ is the same as that for $\operatorname{AWFM}(\breve{f})$ up to time 10 , but then I goes from $(0,-2)$ to $(2,0)$ without going back through his starting point $(0,0)$. For this reason we call $(\tilde{f}, \tilde{g})$ the Nearly Alternating Wait 
For Mommy (NAWFM) strategy.

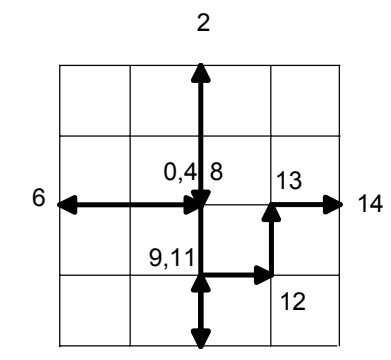

10

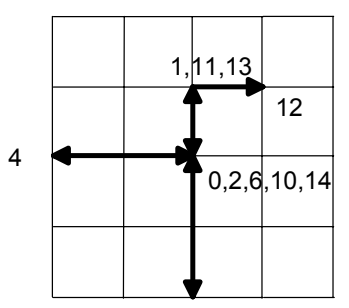

8

(N,N,S,S,W,W,E,E,S,S,N,E,N,E) (N,S,W,W,E,E,S,S,N,N,N,E,W,S)

Figure 4: The NAWFM strategy $(\tilde{f}, \tilde{g})$

The expected meeting time for the NAWFM strategy pair $(\tilde{f}, \tilde{g})$ can be evaluated by consider the following table of $\omega_{i, j, k}(\tilde{f}, \tilde{g})$ and setting $x_{t}$ to be the number of $t$ 's found in the table. The entries $\omega_{i, j, k}$ in the following table are calculated as in the earlier simpler table (12) which was based on the strategy of Figure 1.

\begin{tabular}{|c||c|c|c|c||c|c|c|c|}
\hline \multicolumn{1}{|c||}{$\boldsymbol{\omega}_{i, j, k}(\tilde{f}, \tilde{g})$} & \multicolumn{3}{c||}{$k=$} & \multicolumn{4}{c|}{$k=$} \\
\hline$j$ & 0 & 1 & 2 & 3 & 0 & 1 & 2 & 3 \\
\hline \hline$i=1,(0,2)$ & 2 & 1 & 1 & 2 & 2 & 2 & 1 & 2 \\
\hline$i=2,(2,0)$ & 4 & 8 & 12 & 13 & 14 & 8 & 4 & 12 \\
\hline$i=3,(0,-2)$ & 10 & 4 & 8 & 10 & 10 & 10 & 8 & 4 \\
\hline$i=4,(-2,0)$ & 6 & 6 & 4 & 6 & 4 & 6 & 6 & 6 \\
\hline
\end{tabular}

This gives the agent number profile of

$$
x=x(\tilde{f}, \tilde{g})=[2,6,0,6,0,6,0,4,0,4,0,2,1,1]
$$

(e.g., the are six 2's, so $x_{2}=6$ ) and hence by (14) or (16),

$$
T(\tilde{f}, \tilde{g})=\frac{197}{32} .
$$

\subsection{Nodes with multiple agents}

It will turn out from our subsequent analysis that optimal strategies necessarily involve some simultaneous meetings of Player I with two or more agents of Player 
II. Since this necessitates several agents being simultaneously at a common node, we now analyze how this can occur (both for agents from the same starting node and from different starting nodes).

Lemma 7 (same starting point) Suppose that there are $m>1$ distinct agents from a common starting node $v$ who are all at the same node $z \neq v$ at the same time $t$. Then

(i) $m=2$ and the agents have distinct notions of clockwise.

(ii) The line between $v$ and $z$ makes an angle $r \pi / 4$ with the vertical, $r \in$ $\{0,1,2,3\}$.

(iii) If $r$ is odd, then the two agents have distinct notions of North (distinct $j$ ) and will move to distinct locations at the next move.

Proof. (i) The four rotations $\mathcal{R}^{j}$ take any non-zero vector into four distinct vectors, so agents at the same location must have different notions of clockwise, and hence there can be at most two of them.

(ii) By part (i), the $m=2$ agents at $z$ have distinct notions of clockwise $(k$ equal to 0 and 1 ) and so by (8) have respective locations

$$
v+\mathcal{R}^{j}(g(t)) \text { and } v+\phi \mathcal{R}^{j^{\prime}}(g(t)) \text {, both equal to } z \text {. }
$$

(Recall that $\phi$ is the reflection $\left(w_{1}, w_{2}\right) \rightarrow\left(-w_{1}, w_{2}\right)$ about the vertical axis, and $\mathcal{R}^{j}$ is the clockwise rotation by $\left.j \pi / 4, j=0,1,2,3\right)$. Consequently both $\mathcal{R}^{j}$ and $\phi \mathcal{R}^{j^{\prime}}$ take $g(t)$ into $w=z-v$, and hence $w$ is a non-zero fixed point of $\left(\phi \mathcal{R}^{j^{\prime}}\right)\left(\mathcal{R}^{j}\right)^{-1}=\phi \mathcal{R}^{j^{\prime}-j}=\phi \mathcal{R}^{n}, n=j^{\prime}-j$. If $n=0, w$ lies on the vertical axis (invariant set for $\phi$ ), so $r=0$; if $n=1, \phi \mathcal{R}^{n}\left(w_{1}, w_{2}\right)=\left(-w_{2},-w_{1}\right)$, so the fixed points form the line $w_{2}=-w_{1}$, an angle $3 \pi / 4$ with the vertical; if $n=2$, then $\phi \mathcal{R}^{n}(w)=\left(w_{1},-w_{2}\right)$ with the fixed point line $w_{2}=-w_{2}$ (the horizontal axis), so $r=2$; if $n=3$, then the fixed set $w$ of $\phi \mathcal{R}^{n}$ is the line $w_{1}=w_{2}$, so $r=1$. Note that in all cases, $n$ and $r$ have the same parity.

(iii) By the previous remark, if $r$ is odd, then $n=j^{\prime}-j$ is odd, so the rotations $\mathcal{R}^{j}$ and $\mathcal{R}^{j^{\prime}}$ are not the same or opposite $\left(j \neq-j^{\prime}\right)$, and hence the moves of the two agents (namely $\mathcal{R}^{j}(g(t+1)-g(t))$ and $\left.\phi \mathcal{R}^{j^{\prime}}(g(t+1)-g(t))\right)$ will be distinct, in fact at right angles to each other. (This can also be seen from Figure 1 , which shows that two such agents will have no direction notion in common.)

Lemma 8 (different starting points) Suppose that agents from $m>1$ distinct starting points are simultaneously at a common node $z$. Then

(i) If $m=2, z$ lies on the (Euclidean) perpendicular bisector $B(a, b)$ of the two points $a, b$.

(ii) If $m>2, z$ is the origin $(0,0)$. 
Proof. The vectors $z-v$, for the distinct starting points $v$, are equivalent under rotations, and in particular all have the same Euclidean length (moreover, the set of the absolute values of their two coordinate are the same). Since the Euclidean equidistant set of two points $a, b$ at even lattice distance is their perpendicular bisector $B(a, b)$, this gives (i). The only point equidistant from three starting points is the origin (in either metric), giving (ii).

Lemma 9 There can be at most two agents at a common location $z$ at any odd time $t$. Hence $x_{t} \leq 2$ for odd $t$.

Proof. Assume $x_{t} \geq 3$. Since $t$ is odd, $z$ has odd parity, and hence $z \neq(0,0)$. Hence by Lemma 8 (ii), the agents come from at most two distinct starting nodes $a, b \in \mathcal{S}$. Since by Lemma 7 (i) at most two agents can come from a single starting point, we conclude that $a \neq b$ and $x_{t} \leq 4$. By Lemma 8 (i), $z$ belongs to the perpendicular bisector $B(a, b)$ of $a$ and $b$, which can only contain odd parity nodes $z$ if $a=-b$, and either $z_{1}$ or $z_{2}$ is 0 . Without loss of generality we may assume $a=(2,0), b=(-2,0)$, and $z_{1}=0$. Since by Lemma 7 (ii) the vectors $z-a$ and $z-b$ make angles $r \pi / 4$ with the vertical, the coordinate $z_{2}$ must be 2 , 0 , or -2 , but all these possibilities result in an even parity $z$, whereas $z$ is odd, contradicting the assumption.

\subsection{Starting point meetings (SPM's)}

Lemma 7 (i) says that the only place to simultaneously meet more than two agents from a common starting node $v$ is at $v$ itself. Consequently meetings of this type are important and will be given a special name.

Definition 10 We say there is a Starting Point Meeting (SPM) at time $t$ and node $z$, if either I meets an agent of II at $z=(0,0)$ (called Type I) or Player I meets an agent starting at $z \in \mathcal{S}$ at that node $z$ (called Type II). We denote this (either case) as $\boldsymbol{S P M}(\boldsymbol{t})$.

Note that the Type of the SPM is the name of the Player who is back at his starting point at the time of the meeting. Some elementary properties of SPM's are given below.

\section{Lemma 11 (Starting Point Meetings)}

(i) $\operatorname{SPM}(t)$ implies $t$ is even, $x_{t+1}=0$, and $x_{t} \leq 6$.

(ii) $x_{t}>4$ implies $S P M(t)$, and hence for even $t, x_{t}+x_{t+1} \leq 6$ (so $x_{i} \leq 6$ for all i).

(iii) $x_{t}+x_{t+1} \leq 4$ if no $S P M$ at even time $t$.

(iv) If $S P M(t)$ and $S P M(t+2)$, then the corresponding Types are distinct. 
Proof. (i) Since an SPM must occur at a node $z$ in $\mathcal{S}_{0}$, which contains only even nodes, the time $t$ must be even. Note that at an SPM, the distance between the Players (between I and unmet agents of II) is at least 4, so the earliest time for the next meeting is $t+2$, and hence $x_{t+1}=0$. Suppose the SPM is of type II. At time $t-1$, Player I will be at some node $z^{\prime}$ adjacent to $z$, and there will also be exactly two agents (counting both met and unmet agents) at $z^{\prime}$ from the starting point $z$. Since there are only 8 agents who start at $z$, I can meet at most $8-2=6$ new agents at time $t$ at $z$. If the SPM is of type I, there will be 8 agents at the origin at time $t$ (including ones already met) since $g(t)$ will be a rotation of $(2,0)$. As in the previous argument, I will have met two of them on the previous move. So in either case we have $x_{t} \leq 6$.

(ii) Note that if $x_{t} \leq 4, t$ even, we have $x_{t}+x_{t+1} \leq 4+x_{t+1} \leq 6$ by Lemma 9. So we may suppose that at some time $t$, Player I meets $x_{t}>4$ agents of II (so $t$ is even by Lemma 9) at node $z$. If the first part of (ii) is true, the second part (the 'hence') follows from $x_{t}+x_{t+1} \leq 6+0=6$, by part (i) and Lemma 9 . So we need only prove the first part.

So suppose I meets $x_{t}>4$ agents at some location $z$ at some (even) time $t$, and there is no SPM. So $z \neq(0,0)$ (otherwise we have a Type I SPM). Suppose $z \notin \mathcal{S}$. Since at most two agents can come from any starting point (Lemma $7 \mathrm{i}$ ), the agents met at time $t$ must come from at least three distinct starting nodes, and hence (Lemma 8 (ii)) $z=(0,0)$, again contradicting our assumption. So we may assume that $z \in \mathcal{S}$, and II is not back at his starting point (otherwise we have a Type II SPM). Since we know $z \neq(0,0)$, Lemma 8 (ii) says I can meet agents from at most two starting points. Since II is not back at his start at time $t$, Lemma 7 (i) says there are at most two from each, so in total at most 4 , contradicting the assumption $x_{t}>4$, and we are done.

(iii) Assuming there is no SPM at time $t$, part (ii) implies that $x_{t} \leq 4$. If $x_{t} \leq 2$, the result follows from Lemma 9 (without any other assumptions), so we can assume that $x_{t} \geq 3$. Hence by Lemma 7 (i), agents from at least two starting points $a$ and $b$ must be met at time $t$ at a node $z$, and by Lemma 8 (i), $z \in B(a, b)$.

Suppose $z \in \mathcal{S}$, in which case we can assume without loss of generality that $z=(0,2)$, with $a=(2,0)$ and $b=(-2,0)$. In this case $g(t)$ is a rotation of $(2,2)$, which means the unmet agents at time $t$ are at lattice distance 4 from $z$ and cannot be met before time $t+2$. So $x_{t}+x_{t+1} \leq x_{t}+0 \leq 4$, as required.

Suppose $z \notin \mathcal{S}$. Since $z \neq(0,0)$ (this would be an SPM), we can assume that I meets two agents from $a$ and at least one from $b$. By Lemma 7 (ii), we know that $z-a$ makes an angle $r \pi / 4$ with the vertical. If $a=-b$ ('opposite' starting points) then the only nodes $z$ with this property on $B(a, b)$ are in $\mathcal{S}_{0}$, which we have ruled out. So assume without loss of generality that $a=(2,0)$ and $b=(0,2)$, in which case the only $z$ 's in $B(a, b)$ with the required angle property are $(1,1)$ and $(2,2)$. If $z=(2,2)$ then $g(t)$ is a rotation of $(2,0)$, and the same observation as in the previous paragraph gives $x_{t+1}=0$, and we are done. If $z=(1,1)$, then $g(t)=(1,1)$ and there will be four met and unmet agents at $z$ at time $t$. However by Lemma 7 (iii) these agents will occupy all four nodes adjacent to $z$ at time $t-1$, and again at time $t+1$. By the $t-1$ 
observation, we have $x_{t} \leq 3$, and by the $t+1$ observation and Lemma 9 we know that of the maximum of two agents at I's location $z^{\prime}$ at $t+1$, one has already been met, so $x_{t+1} \leq 1$. Hence $x_{t}+x_{t+1} \leq 3+1=4$, as required.

(iv) If $\operatorname{SPM}(\mathrm{t})$ is of Type II and occurs at some $v \in \mathcal{S}$, then $\operatorname{SPM}(\mathrm{t}+2)$ cannot occur at another $v^{\prime} \in \mathcal{S}$ (also be of Type II), because $d\left(v, v^{\prime}\right)=4$. If $\operatorname{SPM}(\mathrm{t})$ is of Type I, then at time $t$, all unmet agents are at distance 4 from the origin, and hence cannot get there for another Type I meeting before time $t+4$. So consecutive SPM's cannot be of the same type.

Lemma 12 If $S P M(t)$ but not $S P M(t+2)$, then $x_{t+2} \leq 2$ and $x_{t+2}+x_{t+3} \leq 3$.

Proof. Since $\operatorname{SPM}(t)$, one of the players is at his start at time $t$. By renaming them, if necessary, we may assume that Player I is at the origin at time $t$ (Type I). The proof now divides into cases according to the location $z$ of Player I at time $t+2$.

$z=(0,0)$ At time $t$, unmet agents are at distance 4 from $z$, so at time $t+2$ they are at distance at least 2 from $z$, and hence $x_{t+2}=0$. Since $t+3$ is odd, $x_{t+3} \leq 2$ by Lemma 9 and the result follows.

$z \in S$ By symmetry, we may assume $z=(0,2)$. We may assume that at time $t+2$ Player II is not at his starting point $(g(t+2) \neq(0,0))$, since otherwise we have $\operatorname{SPM}(t+2)$. If $x_{t+2} \leq 1$ the result follows from Lemma 9 so we may assume $x_{t+2} \geq 2$. The agents met at time $t+2$ must have starting points $(-2,0)$ or $(2,0)$, since those from $(0,-2)$ were at distance at least 4 from $z$ at time $t$. The agents met at time $t+2$ must be at locations $(-2,2)$ and $(2,2)$ at time $t$. Although there may be two agents at each of these nodes at time $t$, at most one of these (from each) can get to $z$ by time $t+2$, since they have different notions of clockwise. Thus $x_{t+2} \leq 2$. In this case we have $g(t+2)=(2,2)$ so all unmet agents at time $t+2$ are at distance from $z$ of at least 4 , and cannot be met at time $t+3$. Hence $x_{t+2}+x_{t+3} \leq 2$.

$z=( \pm 1, \pm 1)$ By symmetry we take $z=(1,1)$. It is easy to check that the only new agents met at time $t+2$ were at $(2,2)$ at time $t$. There are at most four of these, two each starting from $(0,2)$ and $(2,0)$. Those from each starting point have the same or opposite notion of direction (same North-South axis) but different notions of clockwise, and since $g(t+2)$ is a rotation of $(1,1)$, they will be at different locations at time $t+2$. Hence at most one from each of $(0,2)$ and $(2,0)$ will meet I at $(1,1)$ at time $t+2$, and so $x_{t+2} \leq 2$. If $x_{t+2}<2$ then the result follows from Lemma 9. Assuming $x_{t+2}=2$, the situation is as stated above, and the only nodes with unmet agents within distance 2 of $(1,1)$ at time $t+2$ are $(3,1),(-1,1),(1,3)$, and $(1,-1)$, that is, the four starting points added to $(1,1)$. Player I can meet agents from only one of these nodes at time $t+3$, at respective nodes $z^{\prime}=(2,1),(0,1),(1,2)$, and $(1,0)$. If $z^{\prime}$ is $(2,1)$ (the analysis for $(1,2)$ is similar) the only agents there come from $w=(3,1)$ 
at $t+2$ and (by Lemma 7 (iii)) go to different locations (namely $z^{\prime}$ and $(3,0))$ at time $t+3$. So in this case $x_{t+3}=1$ and we are done. If $z^{\prime}$ is $(0,1)$ (the case $(1,0)$ is similar) then the agents he meets there were at $w=(-1,1)$ at time $t+2$, and they came from at most two starting points $((-2,0)$ and $(0,2))$. If one of these agents goes to $z^{\prime}$, then the other goes to $(-1,0)$.

\section{Optimal strategies}

In this section we first obtain a partial characterization of optimal strategies, then show that the NAWFM strategy $(\tilde{f}, \tilde{g})$ is optimal, and finally give a complete characterization of optimality. We can always rename the players so that the first SPM, if there are any, is of Type II. We do this now. The following important result determines the existence and Type of the potential SPM's at all even times except for $t=14$. We shall see later that optimal strategies may or may not have an SPM at time 14 (if so, it will be of Type II).

Theorem 13 Any optimal strategy pair in $\Gamma(2)$ has SPM's of alternating Types II, I, II, I, II, at times $2,4,6,8$, and 10, and no SPM at time 12.

Proof. To any meeting profile $\left[x_{1}, x_{2}, \ldots\right]$, where $x_{t}$ denotes the number of new agents met at time $t$, we associate the integer $T^{*}=\sum_{t} t x_{t}$, which is 32 times the expected meeting time. To aid the reader, we will highlight the numbers $x_{t}$ in the profile relevant to the argument by grouping them with $\mathrm{a} \sim$. The NAWFM strategy has the agent number profile $[2,6,0,6,0,6,0,4,0,4,0,2,1,1]$, for which $T^{*}=197$ (expected meeting time $197 / 32$ ) and hence any optimal strategy has $T^{*} \leq 197$. We will establish the SPM claims successively for $t=2,4,6,8,10$, and 12 , by rejecting as optimal any strategy which produces a partial agent number sequence $\left[x_{1}, \ldots, x_{k}\right]$ for which the smallest possible value of $T^{*}$ exceeds 197 . Note that for any strategy we have $x_{1}=2$.

$\operatorname{SPM}(2)$ Suppose that $x_{2}+x_{3} \leq 4$. Then by Lemma 11 (ii) the meeting profile minimizing $T^{*}$ is $[2, \overbrace{4,0}, 6,0,6,0,6,0,6,0,2]$, with $T^{*}=202>197$. Hence any optimal strategy has $x_{2}+x_{3}>4$, which by Lemma 11 (iii) implies the required result $\operatorname{SPM}(2)$.

$\operatorname{SPM}(4)$ If there is no SPM at time 4, then by Lemma 12 and the established result $\operatorname{SPM}(2)$, we have $x_{4}+x_{5} \leq 3$. So by Lemma 11 (ii), the minimizing profile is $[2,6,0, \overbrace{3,0}, 6,0,6,0,6,0,3]$, with $T^{*}=206>197$, contradicting optimality. 
$\operatorname{SPM}(6)$ If there is no SPM at time 6, then by $\operatorname{SPM}(4)$ and Lemma 12, we have $x_{6}+x_{7} \leq 3$. Hence by Lemma 11 (ii), the minimizing profile is $[2,6,0,6,0, \overbrace{3,0}^{3}, 6,0,6,0,3]$, with $T^{*}=200>197$.

We now summarize our findings for $t \leq 7$. Since the SPM's at $t=2,4,6$ are of alternating type, it is easy to verify that the meeting profile for any optimal strategy starts with

$$
\begin{aligned}
& {[2,6,0,6,0,6,0], \text { with contribution } T^{* *} \text { to } T^{*} \text { of }} \\
& T^{* *}=(2 \times 1)+(6 \times 2)+(6 \times 4)+(6 \times 6)=74 .
\end{aligned}
$$

Furthermore, there are precisely two starting nodes $a, b \in \mathcal{S}$, which have not been Type II SPM's (not been visited by I) by time 6 . From each of these nodes, two agents have been met at time 4 (the Type I SPM at the origin), and the remaining six agents have not been met by time 6 .

Suppose $x_{8}+x_{9} \leq 2$. Then by (21) and Lemma 11 (ii), the best profile is $[2,6,0,6,0,6,0,2,0,6,0,4]$, with $T^{*}=198>197$. Hence

$$
\begin{aligned}
x_{8}+x_{9} & \geq 3, \text { and }\left(\text { since } x_{9} \leq 2 \text { by Lemma } 9\right) \\
x_{8} & >0 .
\end{aligned}
$$

We now continue to establish further SPM's.

SPM(8) Suppose there is no SPM at time 8. Then by the established SPM at time 6 and Lemma 12, we have $x_{8} \leq 2$ and $x_{8}+x_{9} \leq 3$. Hence by (23)

$$
x_{8}+x_{9}=3 \text { and } x_{9} \geq 1 .
$$

If we also have $x_{10}+x_{11} \leq 4$, then $[2,6,0,6,0,6,0, \overbrace{2,1}, \overbrace{4,0}, 5]$ is the best profile, with $T^{*}=74+125=199>197$. Hence we have $x_{10}+x_{11} \geq 5$. So by Lemma 11 (iii) we have SPM(10), and (Lemma 11 (i)), that $x_{11}=0$. Hence $x_{10} \geq 5$. The SPM at time 10 cannot be Type I, since in that case Player I could meet at most two agents each from the unvisited nodes $a$ and $b$, contradicting the result $x_{10} \geq 5$. Thus it is of Type II, with Player I meeting at least 5 agents at the node (say) $a$. Since there were six agents from $a$ unmet at time 7 , he can have met at most $6-5=1$ of these at times 8 and 9. Since (25) $x_{9} \geq 1$ and the only agents that Player I can meet at time 9 come from $a$ (since they are back at $a$ at the Type II SPM at time 10), we must have $x_{9}=1$ and hence by (25), $x_{8}=2$. Thus the other agent met by I at some node $c$ at time 8 must come from $b$. Since the SPM at time 6 was of Type II, this agent was back at $b$ then, so $d(b, c) \leq 8-6=2$. Now Player I goes from some node $e \in \mathcal{S}$ at time 6 (since there was a Type II SPM then), to $c$ at time 8 , and to $a \in \mathcal{S}$ at time 10. Since $d(e, a)=4$, it follows that the distance from $c$ to all three nodes $a, b, e \in \mathcal{S}$ is no more than 2. Hence $c$ must be the origin, and since I met an agent at $c$ at time 8, there was an SPM at time 8, contradicting our initial assumption. 
By alternation of Types for consecutive SPM's, The SPM at time 8 must be of type I, so at most two agents from each of the unvisited starting points $a$ and $b$ can be met at time 8 , and (by Lemma 11(i)) none at time $9, x_{8} \leq 4$ and $x_{9}=0$.

SPM(10) Suppose there is no SPM at time 10. Then, since we have established $\operatorname{SPM}(8)$, it follows from Lemma 12 that $x_{10} \leq 2$ and $x_{10}+x_{11} \leq 3$. If $x_{8}+x_{9} \leq 3$, it follows from the previous remark and (21), that the best profile satisfying the known constraints is $[\overbrace{2,6,0,6,0,6,0}, \overbrace{3,0}, \overbrace{2,1}, 6]$. But this has $T^{*}=201>197$, so $x_{8}+x_{9} \geq 4$, and by the remarks above this paragraph $x_{8}=4$. Thus, after time 8 , I can meet at most four agents at any SPM of type II, and hence (by Lemma 7 (i)), at most four agents at any time after 8 . Hence the best profile is $[\overbrace{2,6,0,6,0,6,0}, \overbrace{4,0}, \overbrace{2,1}, 4,1]$, with $T^{*}=198>197$. Hence there is an SPM at time 10, which must be of Type II, at node (say) $a$.

Not $\operatorname{SPM(12)~Suppose~there~is~an~SPM~at~time~12,~which~by~alternation~}$ would have to be of Type I. By Lemma 7 (i), I can meet at most two agents from the sole unvisited node $b$ at time 12, so $x_{12} \leq 2$ and (by Lemma 11(i) ) $x_{13}=0$. Hence the best profile would be $[2,6,0,6,0,6,0,4,0,4,0,2,0,2]$, with $T^{*}=198>197$. So there is no SPM at time 12 .

We can now use the pattern of Starting Point Meetings established for optimal strategies in Theorem 13 to determine the unique optimal agent number profile and thus the rendezvous value of $\Gamma(2)$.

Theorem 14 The rendezvous value for $\Gamma(2)$ is 197/32 and the NAWFM strategy $(\tilde{f}, \tilde{g})$ is optimal. Furthermore, every optimal strategy has the agent number profile $[2,6,0,6,0,6,0,4,0,4,0,2,1,1]$.

Proof. Consider an agent number profile $x$ corresponding to an optimal strategy. According to (21) the profile must begin with $[2,6,0,6,0,6,0]$. Theorem 13 says that at time $t=8$, there is a Type I SPM (at the origin), so I will meet there two agents from each of the two starting nodes he has not visited. Hence $x_{8}=4$ and $x_{9}=0$ (by Lemma 11 (i)). At time $t=10$, Theorem 13 says that I will visit his third Player II starting point. Of the eight agents who started there, two were met at the origin at each of the Type I SPM's at $t=4$ and 8 , so $x_{10}=8-2-2=4$, and again $x_{11}=0$. So the agent number profile $x$ must be of the form

$$
\left[2,6,0,6,0,6,0,4,0,4,0, x_{12}, x_{13}, x_{14}\right] .
$$


Since there is no SPM at time 12, it follows from Lemma 4.7 that $x_{12} \leq 2$ and $x_{12}+x_{13} \leq 3$. So the best profile satisfying all known constraints is

$$
[2,6,0,6,0,6,0,4,0,4,0, \overbrace{2,1}, 1],
$$

which is (19) the profile of the NAWFM strategy $(\tilde{f}, \tilde{g})$, and has $T^{*}=197$. Hence NAWFM is optimal, and the rendezvous value is $197 / 32$.

We now use the last two theorems to derive the full set of optimal strategy pairs for $\Gamma$. We describe the situation in I's coordinate system, but we will not use the agent analysis for II. We begin our analysis at the end of move 10 $(t=10)$. According to Theorem 13, I is at one of the previously unvisited nodes of $\mathcal{S}$, say $a$; and II (all four remaining agents of) is at the other one, $b$. Player I can deduce that II is at $b$, while II knows only that the vector to I is one of the four rotations of $(2,2)$. We know from the optimal profile of Theorem 14 that they must meet with conditional probability $1 / 2$ at times 12 (two agents of the remaining 4) and 13 (one agent of the remaining 2); and conditional probability 1 at time 14 (the remaining agent must be met). We will find it easier to use a conditional probability analysis, rather than an agent analysis.

If $a$ and $b$ are opposite starting points $(a=-b)$ in $\mathcal{S}$, then the only possible location for a meeting at time at time 12 is the unique midpoint of $a$ and $b$, namely the origin. But this would constitute an SPM at time 12, which we have ruled out in Theorem 13. The situation from times 10 to 14 is illustrated in Figure 5, with $a=(0,-2)$ and $b=(2,0)$.

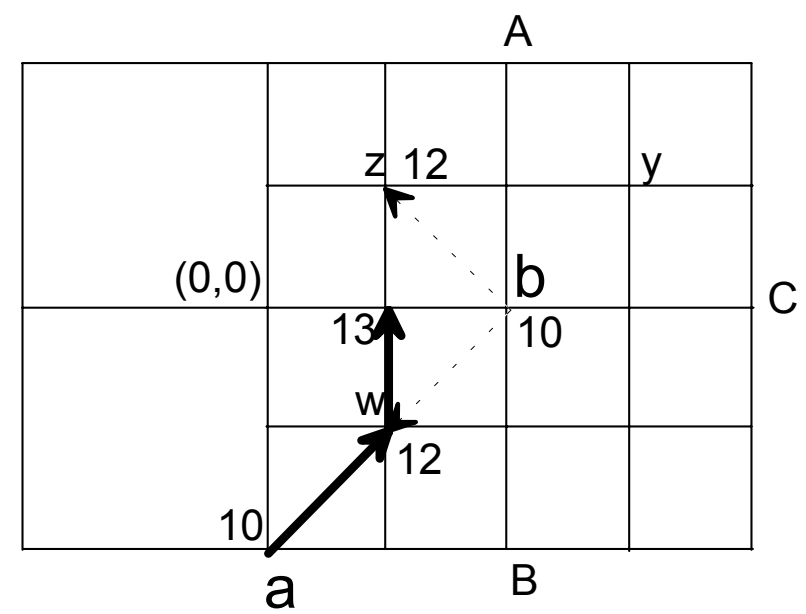

Figure 5: Analysis for $t=10$ to 14 .

Let $w$ denote I's location at time 12 . For a meeting, $w$ has to be at one of the three midpoints of $a$ and $b$ : the origin, $(1,-1),(2,-2)$. The first would imply SPM(12), so can be excluded. The last would require each player to move 2 
units in the same direction on moves 11 and 12 . This would leave the players 4 units apart if they fail to meet at time 12, in which case there could not be a meeting at time 13. So $w$ must be the unique Euclidean midpoint of $a$ and $b$. In our drawing, this gives $w=(1,-1)$. Since the optimal profile has no meeting at time 11, I may get to $w$ in either of the two possible ways (indicated by a diagonal arrow). To achieve a possible meeting at $w$ at time 12, II must move to a diagonally opposite node from his location $b$ at time 10 (must make a turn at time 12). Since he does not know where $w$ is, such a move sequence might lead him to any of the four nodes $w, z, y$, and $(3,-1))$. How can II choose among these four directions so as to reach $w$ at time 12 with conditional probability $1 / 2$ ?

To answer this question, recall (from Theorem 13) that there were Type I SPM's at times 4 and 8. This means Player II has visited two of the possible Player I starting points, that is, two of the three nodes $A, B$, and $C$. (He has not visited the actual starting point at the origin, or the game would be over.) Now there are two cases. Either (1) he did not visit $C$ (visited two opposite starting nodes of I, $A$ and $B$ ) or, (2) he visited $C$ (two adjacent possible starting nodes of I, $C$ and either $A$ or $B$ ). He will know which case he is in, according to whether he searched adjacent or opposite nodes.

Suppose (1) that Player II did not visit $C$, and so visited $A$ and $B$. In this case he can distinguish the vertical coordinate (directions of his searches) from the horizontal (unsearched directions), and for example could determine the origin (I's starting point) with probability $1 / 2$ (he could equiprobably reach the origin or $C$ in two moves). However the node $w$ (which he needs to reach at time 12 with conditional probability $1 / 2$ ) is indistinguishable from three other nodes $z, y$, and $(3,-1)$, and so could not be reached with conditional probability greater than $1 / 4$. Hence he cannot ensure a meeting at time 12 with the required conditional probability $1 / 2$, and so a strategy that $\operatorname{did}$ not visit $C$ cannot be optimal.

So an optimal strategy for II must have visited $C$, that is, previously searched two adjacent possible starting nodes of I. In this case II can determine $w$ with the required conditional probability $1 / 2$ as the node $e$ in the direction opposite the midpoint of the two Player I starting nodes he has visited. (If he visited $A$ and $C$, then $e=w$; if $B$ and $C$, then $e=z$.) Optimal play must bring him to $w$ with conditional probability $1 / 2$, so he must move to $e$ (in either of the two possible ways, since the optimal profile has no meeting at $t=11$ ) at time 12. This is indicated by the dotted line going out from $b$. (This is not a mixed strategy, II goes for his uniquely defined node $e$.) Assuming II does not meet I at time 12 , his location at that time must be $e=z$, and I can deduce this. So to achieve a possible meeting at time 13 (required by the optimal profile), I must move to the unique lattice midpoint of $w$ and $z$ (the node $(1,0)$ in our figure). Player II can conclude at time 12 that Player I is either at $w$ or $y$. So he must move back toward $b$ equiprobably in either way (toward $w$ or toward $y$ ). (Again, this is not a mixed strategy - in agent formulation, one of the two remaining agents will go towards $b$ clockwise, and the other counterclockwise.) If the Players have not met by time 13, they both can deduce the location of the 
other; $\mathrm{I}$ is at $(1,0)$ and II is at $(2,1)$. Now they can agree to meet for sure at time 14 at either of their two midpoints (i) $b$, or (ii) $z$. This leads to two types of strategy, according to whether they both choose (i) or they both choose (ii).

Hence we have established the following:

Theorem 15 A strategy pair is optimal in $\Gamma(2)$ if and only if it is one of the following two types (i) and (ii).

Player $I$ orders the starting points of $I I$ as $V_{1}, V_{2}, V_{3}, V_{4}$ in any way so long as the first two are adjacent $\left(V_{1} \neq-V_{2}\right)$. On the first ten moves, he goes to $V_{1}$ and back, $V_{2}$ and back, and to $V_{3}$. On moves 11 and 12 he goes (in either of the two ways) to the unique Euclidean midpoint $w$ of $V_{3}$ and $V_{4}$. At time 13 he goes to the unique midpoint of the origin, $w$, and $V_{4}$. At time 14 , he either (i) goes to $V_{4}$, or (ii) goes in the same direction as on move 13.

Player II goes in any direction on move 1, returning to his start on move 2. On moves 3 to 6 , and on moves 7 to 10 , he goes respectively to two adjacent possible starting nodes $W_{1}$ and $W_{2}$ and back to his start. On moves 11 and 12 , he goes (in either of the two possible ways) to the node e diagonally opposite his start and in the direction opposite to the Euclidean midpoint of $W_{1}$ and $W_{2}$. On move 13, he goes to either of the two midpoints of e and his start. On move 14, he goes either to (i) his starting point, or (ii) back to e.

The strategy $(\tilde{f}, \tilde{g})$ is of type (i). In Figure 6 we give an example of an optimal strategy of type (ii).

2

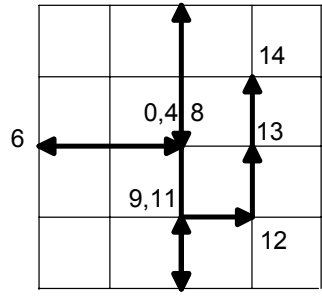

10

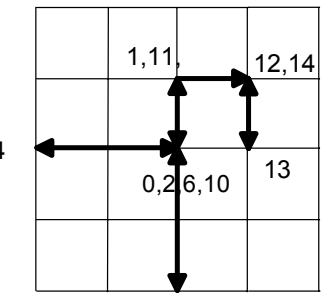

8

\section{$(\mathrm{N}, \mathrm{N}, \mathrm{S}, \mathrm{S}, \mathrm{W}, \mathrm{W}, \mathrm{E}, \mathrm{E}, \mathrm{S}, \mathrm{S}, \mathrm{N}, \mathrm{E}, \mathrm{N}, \mathrm{N}) \quad(\mathrm{N}, \mathrm{S}, \mathrm{W}, \mathrm{W}, \mathrm{E}, \mathrm{E}, \mathrm{S}, \mathrm{S}, \mathrm{N}, \mathrm{N}, \mathrm{N}, \mathrm{E}, \mathrm{S}, \mathrm{N})$}

Figure 6: Optimal strategy for $\Gamma$ of type (ii)

Although the NAWFM strategy is optimal, it is not uniformly optimal - it does not maximize the probability of meeting by time $t$ for all $t$. In particular, it does not maximize the probability of meeting by time 12. After move 12, Player I has met 30 of the 32 agents, so the probability that the Players have 
met by time 12 is $30 / 32$. Consider the 12 move strategy for Player I given by $(N, W, S, W, E, E, S, S, N, N, E, E, \ldots)$, and drawn in Figure 7 .

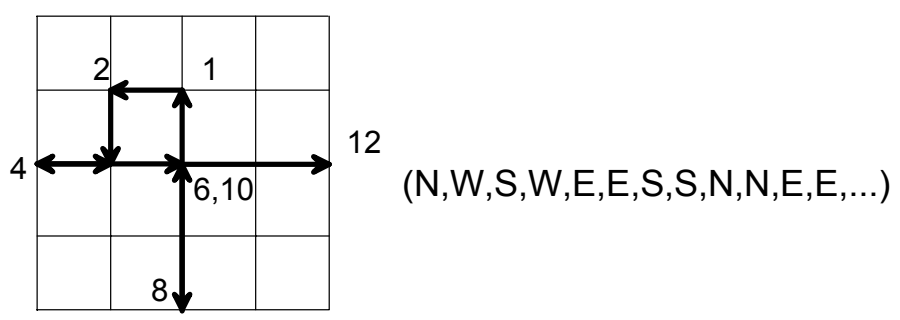

Figure 7: Strategy with meeting probability $31 / 32$ by time 12 .

Assuming Player II is back at his start at times 4, 8, and 12, the only agents not met by time 12 have starting point $(0,2)$. Of the eight agents who started there, two could be met at $(0,1)$ at time 1 , one (only one since this involves a turn) could be met at $(-1,1)$ at time 2 , and two could be met at the origin at both times 6 and 10. This would leave only one agent unmet by time 12 , or a $31 / 32$ probability of rendezvous by that time, better than NAWFM. In fact, the agents can be met at the times mentioned above if Player II adopts the move sequence $(N, E, W, S, S, S, N, N, W, W, E, E, \ldots)$. Hence the NAWFM strategy is optimal but not uniformly optimal and, as mentioned in Definition 4 , there cannot be any uniformly optimal strategy for the game $\Gamma=\Gamma_{P}$. As mentioned in [4], this is qualitatively distinct from the situation for the diagonal start game $\Gamma_{D}$, where there is a uniformly optimal strategy (and hence all optimal strategies are uniformly optimal). The (uniformly) optimal strategies for the diagonal start game (without common clockwise) are as follows (Corollary 23, [4]): Player I cyclically orders adjacent (distance 2) starting nodes as $v_{1}, v_{2}, v_{3}, v_{4}$ in any manner. He moves to the midpoint of $v_{1}$ and $v_{4}$ at time 1 , and then cyclically searchers the vertices $v_{i}$ at time $2 i$. Player II moves in any direction $D_{i}$ (of the four possible) at times $i=1,3,5,7$, going in the opposite direction $-D_{i}$ at times $i+1$. The only restriction is that $D_{7}= \pm D_{1}$, that is, II must move in the same or opposite direction on move 7 as he did on move 1 (not at right angles). The particular strategy pair of $(N, W, S, S, E, E, N, N),(N, S, N, S, N, S, N, S)$ was earlier shown to be optimal for the common clockwise version of the diagonal start problem by Anderson and Fekete [10], and an easy symmetric argument shows that it does equally well (same expected meeting time) in the no common clockwise version.

\section{Rendezvous in Higher Dimensions}

This section determines an upper bound for the rendezvous value $R(n)$ of the parallel start game $\Gamma(n)=\Gamma_{P}(n)$ which generalizes the game $\Gamma(2)$ of the 
previous section (as well as the game $\Gamma(1)$ solved in [7]) to $n$ dimensions. The strategy pair giving this bound is the AWFM (Definition 6), which assumes no common ordering of the coordinate axes. Some ideas on lower bounds are also given at the end of the section. For notational simplicity we will remove the subscript $P$.

Theorem 16 Suppose that two players are initially placed on the $n$-dimensional integer lattice so that their difference vector is two units long and parallel to one of the coordinate axes. Assume that the players have no common notion of location and no common labelling of the coordinates axes. The rendezvous value $R(n)$ for this game $\Gamma(n)$ satisfies the inequality

$$
2 n \leq R(n) \leq \frac{32 n^{3}+12 n^{2}-2 n-3}{12 n^{2}}
$$

Consequently, we have the asymptotic result

$$
2 \leq \lim _{n \rightarrow \infty} R(n) / n \leq \frac{8}{3} .
$$

We will need to consider two subsidiary problems $\Gamma_{1}(m)$ and $\Gamma_{2}(m)$, for $m=1, \ldots, 2 n$. Both of these problems begin at time $t=0$ with the placement of Players I and II respectively at a pair of nodes $A$ and $B$ which are two units apart along a line parallel to some coordinate axis. Then Player I is displaced to a node $A^{\prime}$ along a similar two unit line which is not the one leading to $B$. Player I is told the node $A$. In the problem $\Gamma_{1}(m)$, Player I is told $m-1$ directions which are certain to include the direction to $B$, and Player II is told $m$ such directions. In problem $\Gamma_{2}(m)$, both players are told $m$ such directions. Special cases of these problems, for $n=2$, are drawn in Figures 8 and 9 .

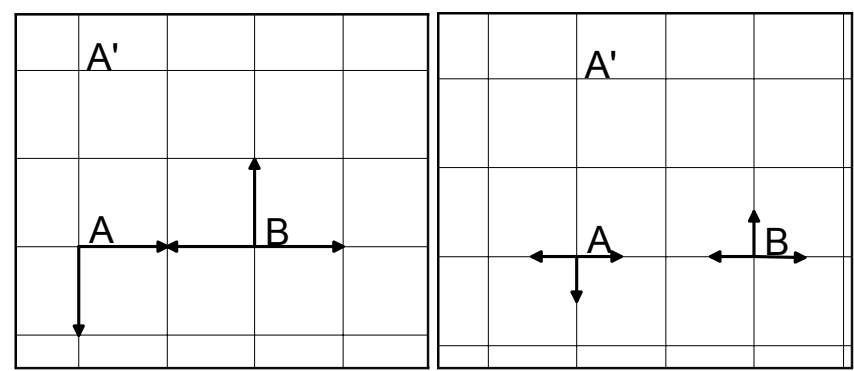

Figure 8: Start in $\Gamma_{1}(3)$. Figure 9: Start in $\Gamma_{2}(3)$.

In order to estimate the rendezvous value $R^{a}(n)$ of the original problem $\Gamma(n)$, we must obtain estimates on the respective asymmetric rendezvous values $w_{1}(m)$ and $w_{2}(m)$ of $\Gamma_{1}(m)$ and $\Gamma_{2}(m)$ for various $m$ (corresponding to the dimension $n$, which is implicit in our notation).

Suppose that in the problem $\Gamma_{1}(m)$, the first two moves of the players are as follows: Player I goes to the node $A$ (which he knows) while Player II goes 2 steps randomly in one of the $m$ indicated directions. With probability $1 / m$, II 
will pick the direction to $A$, and the meeting time will be $t=2$. Otherwise the two players will be in the initial position of the other problem $\Gamma_{2}(m-1)$, with the roles (of I and II) reversed. Hence we have

$$
w_{1}(m) \leq \frac{1}{m}(2)+\frac{m-1}{m}\left(2+w_{2}(m-1)\right) .
$$

Similarly, in the initial position of $\Gamma_{2}(m)$, the same type of strategy for the first two moves gives

$$
w_{2}(m) \leq \frac{1}{m}(2)+\frac{m-1}{m}\left(2+w_{1}(m)\right) .
$$

From these two inequalities we obtain upper bounds $w_{i}(m) \leq \bar{w}_{i}(m)$ by solving the associated equalities. This gives us the solutions

$$
\begin{aligned}
& \bar{w}_{1}(m)=\frac{4 m+1}{3}, \\
& \bar{w}_{2}(m)=\frac{-1+3 m+4 m^{2}}{3 m} .
\end{aligned}
$$

This is consistent with the trivial base case $\Gamma_{2}(1)$, where I and II start 4 units apart with knowledge of the other's direction, with rendezvous value $w_{2}(1)=2$ corresponding to a meeting at $A$. This case is illustrated in Figure 10.

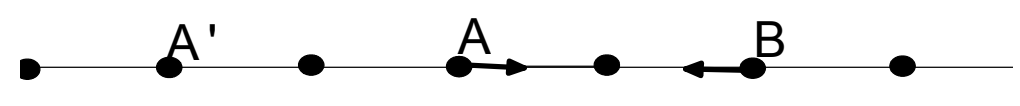

Figure 10: Start position in $\Gamma_{2}(1)$.

We now consider the original game $\Gamma(n)$. Suppose that in their first two moves one player (I) goes two units in his forward direction, while the other (II) goes in some direction and then back to his start. With probability $1 /(2 n)^{2}$ the two players will go towards each other and meet at time $t=1$. If Player I goes in the direction of II (probability $1 / 2 n$ ) and II does not go in the direction of I (probability $(2 n-1) /(2 n))$ then the two will meet at time $t=2$ at II's initial location. In the remaining case, I will find himself displaced two units from his start and know of one direction from his start which does not lead to II's starting location. Meanwhile, II will be at his start and not know of any of the $2 n$ directions which are not correct. Consequently the situation at time $t=2$ will be identical to that of the problem $\Gamma_{1}(2 n)$. Therefore we have the estimate

$$
R(n) \leq\left(\frac{1}{(2 n)^{2}}\right) 1+\left(\frac{1}{2 n}\right)\left(\frac{2 n-1}{2 n}\right) 2+\left(\frac{2 n-1}{2 n}\right)\left(2+w_{1}(2 n)\right) .
$$


Estimating $w_{1}$ by the formula (29) for $\bar{w}_{1}$, and simplifying, we get

$$
\begin{aligned}
R(n) & \leq \frac{32 n^{3}+12 n^{2}-2 n-3}{12 n^{2}}, \text { and hence } \\
\lim _{n \rightarrow \infty} R(n) / n & \leq \frac{8}{3}, \text { the right inequality in Theorem } 27 .
\end{aligned}
$$

We note again that the value of the right hand side of the top inequality is $13 / 4$ for $n=1$, which is the exact asymmetric rendezvous value for the line as derived in [7] for known initial distance $D=2$. The strategy pair which gives this expected meeting time is the one which converts $\Gamma(n)$ into $\Gamma_{1}(2 n)$ and thereafter converts each problem $\Gamma_{1}(m)$ into $\Gamma_{2}(m-1)$ and each problem $\Gamma_{2}(m)$ into $\Gamma_{1}(m), m=2 n, 2 n-1, \ldots, 1$ (assuming the players don't meet earlier). It is an AWFM strategy.

We can obtain a lower bound on $R(n)$ by giving the players some additional information and determining their optimal strategy pair in that situation. The simplest way to do this is to give the players common notions of directions. In this case it is well known (see [7] or [10] for one or two dimensional arguments) that the players should always move in opposite directions, and that the rendezvous problem is consequently equivalent to one where Player II is stationary and Player I moves with their combined speeds, here equal to 2 . The least expected time for Player I to reach the $2 n$ possible locations of the stationary player II, moving with speed 2 , is obtained by a path which reaches these locations at the $2 n$ times $1,3,5, \ldots, 2 i-1, \ldots, 2(2 n)-1$, while returning to his start in between. The expected time is consequently given by

$$
\frac{1}{2 n} \sum_{i=1}^{2 n}(2 i-1)=2 n \text {. }
$$

Thus the asymptotic value of $R^{a}(n) / n$ lies between 2 and $8 / 3$, completing the proof of Theorem 27. A player-symmetric version of this $n$-dimensional problem, where both players must follow the same mixed strategy, is analyzed in [8] (Section 18.2.2). An alternate treatment of the player-asymmetric problem is outlined in Section 18.2.1. 


\section{References}

[1] Alpern, S. (1995). The rendezvous search problem. SIAM J. Control \& Optimization 33, 673-683.

[2] Alpern, S. (2002a). Rendezvous search: A personal perspective. Operations Research 50, No. 5, 772-795.

[3] Alpern, S. (2002b). Rendezvous search on labelled graphs. Naval Research Logistics 49, 256-274.

[4] Alpern, S., and Baston, V. (2004). Rendezvous on a Planar Lattice. CDAM Research Report Series, Centre for Discrete and Applicable Mathematics, LSE 2004-8.

[5] Alpern, S., and Baston, V. (2004). A common notion of clockwise helps in planar rendezvous. Rendezvous on a Planar Lattice. CDAM Research Report Series, Centre for Discrete \& Applicable Mathematics, LSE 2004-7.

[6] Alpern, S., and Beck, A. (1999). Asymmetric rendezvous on the line is a double linear search problem. Math. Operations Research 24, no. 3, 604618 .

[7] Alpern, S., and Gal, S. (1995). Rendezvous search on the line with distinguishable players. SIAM J. Control \& Optimization 33, 1270-1276.

[8] Alpern, S., and Gal, S (2003). The theory of search games and rendezvous. International Series in Operations Research and Management Science, Volume 55, Kluwer Academic Publishers, Boston, 336 pp.

[9] Anderson, E. J., and Essegaier, S. (1995). Rendezvous search on the line with indistinguishable players, SIAM Journal of Control and Optimization $33,1637-1642$.

[10] Anderson, E. J., and Fekete, S. (2001). Two dimensional rendezvous search. Operations Research 49, 107-118.

[11] Baston, V. J. (1999). Two rendezvous search problems on the line, Naval Research Logistics 46, 335-340.

[12] Baston, V. J. and Gal, S. (1998). Rendezvous on the line when the players' initial distance is given by an unknown probability distribution. SIAM Journal of Control and Optimization 36, no. 6, 1880-1889.

[13] Gal, S., (1999). Rendezvous search on the line. Operations Research 47, 974-976.

[14] Howard, J. V. (1999). Rendezvous search on the interval and circle. Operations Research 47, No. 4, 550-558.

[15] Thomas, L. C. and Hulme, P. B. . Searching for targets who want to be found. Journal of the O. R. Society 48 (1997), Issue 1, Pages 44 - 50. 